\title{
Estimates of critical thermal environments for mule deer
}

KATHIRINE L. PARKER AND MICHAEL P. GLLINGHAM

\begin{abstract}
Abatract
Mule deer (Odocolleus hemionus hemionus) can be thermally stresced under a wide range of ambient conditions. We developed a model that provides examples of the combinations of wind, solar radiation, and air temperature that may result in thermally critical environments for standing, full-fed adult mule deer during winter in snow-covered and snow-free, open habitats, and in meadows in summer. Critical thermal combinations of environmental variables are shown as 3-dimensional surfaces and tables. Animal size, age, pelage characterbtics, and ground cover (height and albedo) further affect the energy costs for thermoregulation by mule deer. The need for habitat managers to consider the provision of thermal cover to reduce heat or cold stress in mule deer depends on the combinations of environmental variables in a particular habitat and geozraphic location. Implication, limitations, and management comiderations of our extimates are discuased.
\end{abstract}

Key Words: energetics, Odocoileus, standard operative temperature, thermal cover, thermoregulation

Field observations suggest that wild ungulates select cover, bedding sites, and postures for apparent thermal benefits (Beall 1974, Leckenby 1977). Thermal cover now is considered an important component of ungulate habitat (Black et al. 1976), although few studies have quantified the thermal environments experienced by animals and much of the information on movements or habitat selection cannot be used to distinguish between animal preference and requirement. Bioenergetics research on captive animals has identified physiological mechanisms that allow wild ungulates to cope with climatic stress and has defined many of their energetic requirements (e.g., Parker and Robbins 1984, Renecker and Hudson 1986). Increased knowledge of the interactions between weather variables and animal responses is needed if thermal conditions are to be considered in habitat-management (Thomas et al. 1979).

In most studies, air temperature $\left(T_{2}\right)$ alone has been used to describe the thermal environments experienced by animals. Standard operative temperature $\left(T_{\infty}\right)$ (Bakken 1980, 1981), however, more appropriately describes the effective temperature experienced by animals outdoors by integrating effects of air temperature, wind speed, and incident radiation into a single variable describing the animal's thermal environment. Thermal conditions of different habitats for a species may then be compared with a common thermal index. Effects of precipitation, however, are not included in this index.

Thermoregulation is an animal's ability to regulate body temperature within acceptable limits despite large variations in ambient conditions. Over a thermoneutral range of environmental temperatures, an animal's resting metabolic rate remains relatively constant and independent of environmental conditions (e.g., Parker and Robbins 1985). As the animal approaches the lower limit of this thermoneutral zone (TNZ) or lower critical temperature

\footnotetext{
Authors are assistant ecientists, Wildlife Biology Program, Washington State University, Pullman 99164. At the time of the research, both authors were research associates, Faculty of Forestry, University of British Columbia, Vancouver, B.C. V6T IWs.

Research was funded in part by the Science Council of British Columbia through a grant to F.L. Bunnell. The insights and contributions by G.S. Campbell are greatly appreciated. Helpful comments on this manuscript were provided by R.T. Bowyer.

Manuscript accepted 16 March 1989.
}

(LCT), it increases the thermal resistance of the hair coat by piloerection and that of the tissue by peripheral vasoconstriction. When physical regulation of body temperature reaches a maximum, chemical regulation occurs. Energy metabolism must increase to maintain acceptable body temperature and prevent impairment of tissue function. Above the upper limit of the thermoneutral zone, or upper critical temperature (UCT), metabolic rate increases and is usually associated with hyperthermia. To minimize energy costs for thermoregulation by a wildlife species, habitat management could provide thermal cover to ameliorate environmental conditions when standard operative temperatures are less than LCT's and greater than UCT's.

Although the concept of critical thermal environments has already been proposed (Moen 1968a), wildlife managers currently place little emphasis on thermal constraints. Our specific objectives are to: (1) present a model that provides examples of thermally critical environments for mule deer in winter and summer based on standard operative temperature; and (2) describe these environments in tabular and graphical format. With this information, managers will be better able to determine whether thermally critical environments are commonly experienced by mule deer in specific habitat conditions.

\section{Methods and Theory}

To examine the effects of weather on ungulates in the wild, we generated a thermal model to assess physiological responses of mule deer to various combinations of micrometeorological variables (see Appendix 1 for general equations).

\section{Calculation of Operative Temperature}

Thermal environments of mule deer initially were quantified using the equation for operative temperature $\left(T_{\bullet}\right)$, presented by Campbell (1977:94):

$$
T_{0}=T_{a}+\frac{r_{e}\left(R_{\mathrm{abe}}-\epsilon_{\mathrm{a}} \sigma T_{\mathrm{a}}^{4}\right)}{\rho c_{\mathrm{p}}}
$$

where the temperature experienced by the animal $\left(T_{e}\right)$ includes air temperature and the effects of wind and radiation. These effects incorporate $r_{e}$, the animal's resistance to convective and radiative heat transfer; $\mathbf{R}_{\mathrm{aba}}$, the solar and thermal radiation absorbed by the animal; $\epsilon_{+} \sigma T_{a}^{4}$, thermal emittance of the animal's surface at air temperature; and $\rho c_{p}$, the volumetric specific heat of air. Preliminary estimates of $T_{e}$ for mule deer (Parker and Gillingham 1987) incorporated the influence of wind on coat resistance but underestimated the effects of high wind speeds. Therefore, a wind-chill corrected or standard operative temperature $\left(T_{e a}\right)$, based on theory developed by Bakken (1981), was used to better estimate the animal's thermal environment:

$$
T_{e \mathrm{a}}=\frac{T_{\mathrm{b}}-\mathrm{r}_{\mathrm{HBS}}+\mathrm{r}_{\mathrm{ca}} \cdot\left(T_{\mathrm{b}}-T_{0}\right)}{\mathrm{r}_{\mathrm{Hb}}+\mathrm{r}_{\mathrm{b}}}
$$

where $T_{\mathrm{b}}$ is the animal's body temperature, $\mathrm{r}_{\mathrm{Hb}}$ is the thermal resistance of skin and pelage to heat flow under natural outdoor wind speeds $(u)$, and $r_{\mathrm{Hb}_{e}}$ and $r_{\mathrm{Es}}$ are the values of $r_{\mathrm{Hb}}$ and $r_{0}$ under low convective conditions $(u \leq 1 \mathrm{~m} / \mathrm{s})$. 


\section{Physiological Measurementa}

Using the energetic measurements made on animals under outdoor conditions from Parker (unpubl.) and Parker and Robbins (1984), we employed nonlinear regression techniques (Dixon 1981) to describe the relationships between ambient conditions and body temperature, and thermal resistance of mule deer at low wind speeds $(\mathrm{u} \leq 1 \mathrm{~m} / \mathrm{s})$. Animal whole body resistance was partitioned into tissue and coat components. Tissue resistance was assumed to be an inversely linear function between vasoconstriction at lower critical temperature $(170 \mathrm{~s} / \mathrm{m})$ and vasodilation at upper critical temperature $(50 \mathrm{~s} / \mathrm{m})$ (Webster 1974). Coat resistance was calculated as the difference between whole body and tissue resistances. At wind speeds greater than $1 \mathrm{~m} / \mathrm{s}$, we assumed that coat resistance decreased $8 \%$ with each $\mathrm{m} / \mathrm{s}$ increase in wind speed, as noted for caribou, wolf, and rabbit (Campbell et al. 1980).

Adult animals were assumed to weigh $67 \mathrm{~kg}$ (Parker 1983). In winter, LCT was fixed at $-19^{\circ} \mathrm{C}$ and upper temperature was $+2.5^{\circ}$ C (Parker and Robbins 1984:1418). In summer, lower and upper critical limits were $+5^{\circ}$ and $23.5^{\circ} \mathrm{C}$, respectively (Parker and Robbins 1984:1419).

\section{Effects of Wind}

Wind speeds, standardly measured at $3 \mathrm{~m}$ above the ground, were extrapolated to animal height (Campbell 1977:38). This height was assumed to be the height of mid-rib cage, which varies as a function of body weight for mule deer (Parker 1987). The average 'crop' height (grass, shrubs, snow) in which the animal was standing was fixed at $0.1 \mathrm{~m}$ for this analysis. A characteristic dimension, or representative distance over which the wind travels on the animal's surface (Campbell 1977:65) was computed as the average of the animal's length and diameter (Parker 1987).

Effects of convection, forced (resulting directly from wind speed) and free (occurring because of thermal gradients between the animal and its environment), were assessed prior to determining the animal's resistance to convective heat transfer. Usually one or the other process dominates, and we used only the resistance for the dominant process (Campbell 1977:70). Resistance to free convection was calculated from the temperature difference between the air and the animal's skin surface in the sun or shade; this resistance occurred over the characteristic dimension of the animal.

\section{Effects of Solar and Thermal Radiation}

We separated short-wave irradiance into direct and diffuse com-
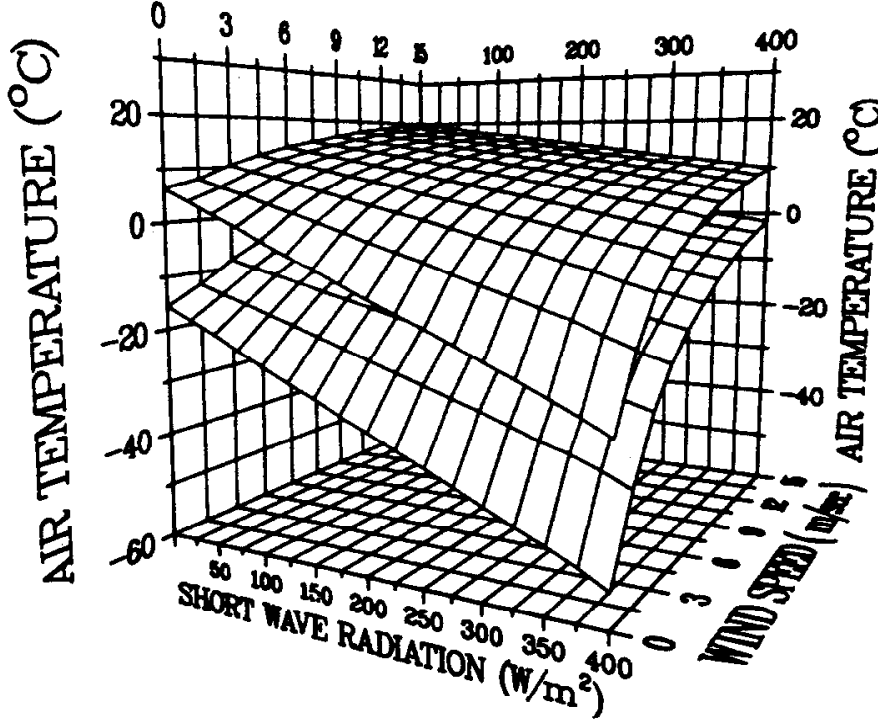

Fig. 1. Three-dimensional surfaces of predicted lower $\left(T_{\mathrm{en}}=49^{\circ} \mathrm{C}\right)$ and upper $\left(T_{e}=2.5^{\circ} \mathrm{C}\right)$ thermally critical environments for full-fed, adult mule deer standing in snow-covered, open habitats in winter.

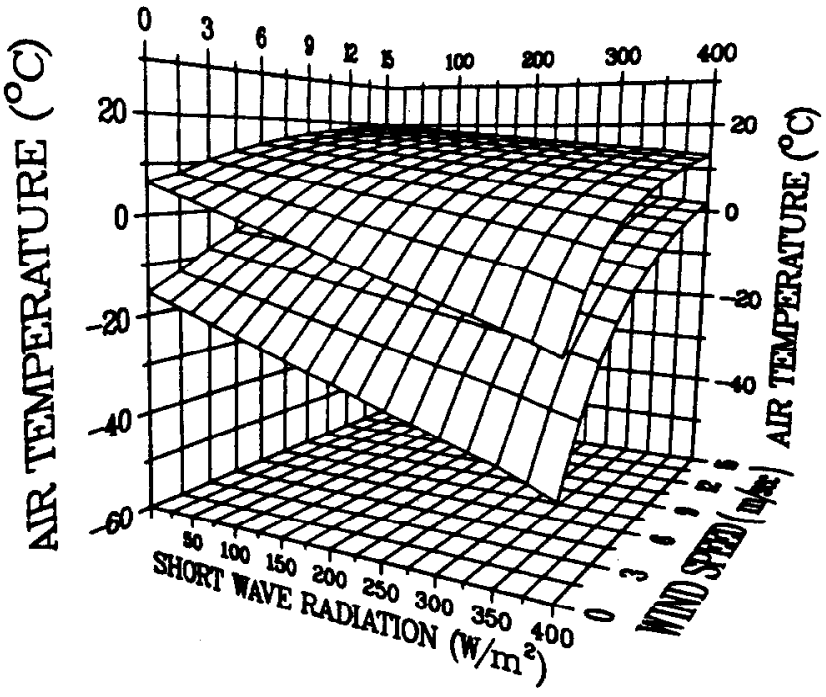

Fig. 2. Three-dimensional surfaces of predicted lower $\left(T_{e=}=-19^{\circ} \mathrm{C}\right)$ and upper $\left(T_{e}=2.5^{\circ} \mathrm{C}\right)$ thermally critical environments for full-fed, adult mule deer standing in snow-free, open habitats in winter.

ponents using factors to incorporate differences between potential and measured direct radiation and the amount of absorption of the solar beam by atmospheric moisture and ozone (Wesley and Lipschutz 1976, Campbell 1981). Reflected short-wave radiation was estimated using surface albed os of 0.80 for snow, 0.20 for a field in summer, and 0.26 for matted grass in winter (Lowry 1969:133). Angle of solar elevation was defined by the interaction among solar declination, geographic latitude, time of day, and time of solar noon (Paltridge and Platt 1976, Campbell 1981). Total short-wave radiation absorbed by the animal was then calculated assuming a shadow area equal to $1 / 3$ of the animal's surface area and shortwave absorptivity values of 0.7 and 0.8 in summer and winter pelage, respectively (W. Porter, pers. comm.).

In the calculation of long-wave radiation absorbed by the animal, we employed a clear sky emissivity based on air temperature (Moen 1968b:340), a ground emissivity of 0.97 (K.L. Parker, unpubl. data), and an animal emissivity of 1.0 , as demonstrated for caribou (Rangifer tarandus) (Monteith 1973). Because of the extreme variability of thermal energy flux from animal surfaces
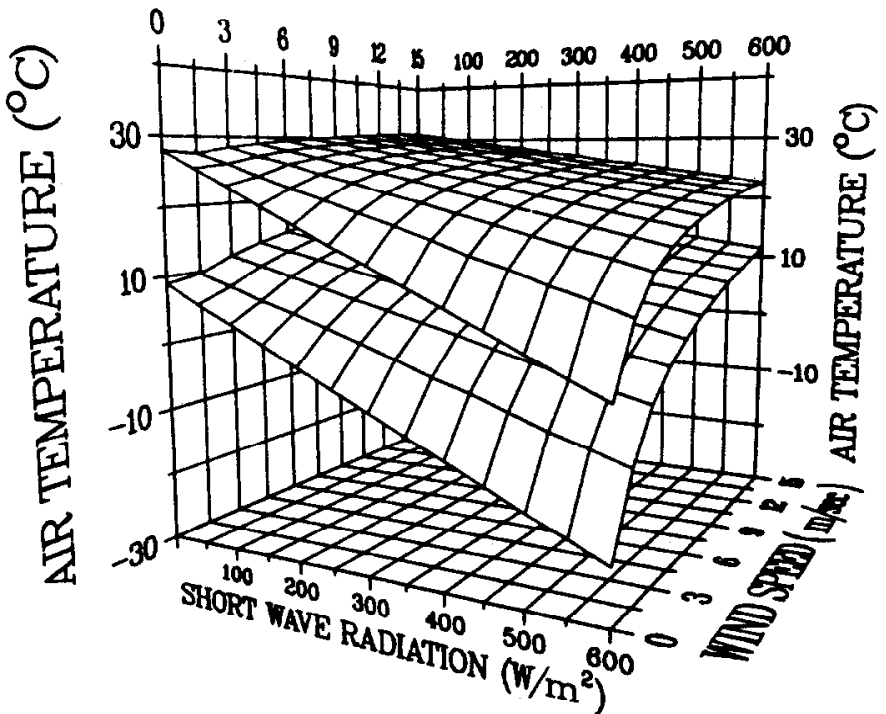

Fis. 3. Three-dimenaional surfaces of predicted lower $\left(T_{e c}=5^{\circ} \mathrm{C}\right)$ and upper $\left(T_{e}=23.5^{\circ} \mathrm{C}\right)$ thermally critical environments for full-fed, adult mule deer standing in meadowed habitats in summer. 
when exposed to ambient conditions (Moen 1973:88, 1974, Moen and Jacobsen 1974) and because these interactions between environmental variables and surface temperatures have not been thoroughly quantified, we assumed that the animal's outer surface temperature equalled air temperature.

\section{Model Inputs and Outputs}

$T_{\infty}$ was determined for combinations of air temperature ( -20 to $+20^{\circ} \mathrm{C}$ in winter, 0 to $40^{\circ} \mathrm{C}$ in summer), wind speed $(0$ to $15 \mathrm{~m} / \mathrm{s})$, and short-wave radiation $\left(0\right.$ to $400 \mathrm{~W} / \mathrm{m}^{2}$ in winter, 0 to $800 \mathrm{~W} / \mathrm{m}^{2}$ in summer) at $46.83^{\circ} \mathrm{N}, 117.20^{\circ} \mathrm{W}$ (Pullman, Washington). Predictions were made under no sun conditions and at a maximum angle of solar elevation (time of day = time of solar noon) in midsummer (Julian day $=200$ ) and midwinter (Julian day $=49$ ) under conditions with and without snow. These predictions and planes of lower and upper critical temperatures are presented in tabular and 3dimensional formats. Graphical output was produced using Dis- spla Integrated Software System and Plotting Language (ISSCO 1987).

\section{Results}

\section{Predicted Standard Operative Temperatures}

Incident solar radiation raises the standard operative temperature ( $\left.T_{\text {en }}\right)$ experienced by an animal and wind speed decreases the effective environment (Tables 1-3). In winter, the high reflectance of solar radiation from snow increases standard operative temperatures up to $9^{\circ} \mathrm{C}$ from those experienced on bare ground at midday under the same air temperature, still air, and high solar level conditions (Table 1,2). Wind speeds of $5 \mathrm{~m} / \mathrm{s}$ can eliminate most of the thermal effects of maximum solar levels. In summer, incident solar levels may be twice as high as those occurring in winter (Table 3). The relative effects of these high solar loads at midday with minimal wind, however, are comparable to those in snow-covered winter habitats and may raise standard operative temperatures $30^{\circ}$

Table 1. Predicted standard operative temperatures $\left(T_{\mathrm{ea}}\right)$ for full-fed, adult mule deer standing in snow-covered open habitats in winter. $T_{a}$ is air temperature; $u$ is wind speed at $3 \mathrm{~m}$; $u_{\mathrm{a}}$ is wind speed at animal height; $S W$ is short-wave radiation.

\begin{tabular}{|c|c|c|c|c|c|c|c|c|c|c|c|c|c|c|}
\hline $\begin{array}{l}T_{a} \\
\left({ }^{\circ} \mathrm{C}\right)\end{array}$ & $\underset{(m / s)}{u}$ & $\begin{array}{c}u_{a_{a}} \\
(m / s)\end{array}$ & $\begin{array}{c}S W \\
\left.W / m^{2}\right)\end{array}$ & $\underset{\left({ }^{\circ} \mathrm{C}\right)}{T_{e}}$ & ${ }_{\left({ }^{\circ} \mathrm{C}\right)}^{T_{n}}$ & $\underset{(m / s)}{u}$ & $\begin{array}{r}u_{\mathrm{n}} \\
(\mathrm{m} / \mathrm{s})\end{array}$ & $\begin{array}{c}S W \\
\left.W / m^{2}\right)\end{array}$ & $\begin{array}{c}T_{\text {ea }} \\
\left({ }^{\circ} \mathrm{C}\right)\end{array}$ & $\begin{array}{r}T_{a} \\
\left({ }^{\circ} \mathrm{C}\right)\end{array}$ & $\underset{(m / s)}{u}$ & $\underset{(\mathrm{m} / \mathrm{S})}{\mathrm{u}_{\mathrm{a}}}$ & $\begin{array}{c}S W \\
\left.W / m^{2}\right)\end{array}$ & $\begin{array}{c}T_{\text {eas }} \\
\left({ }^{\circ} \mathrm{C}\right)\end{array}$ \\
\hline-25 & 0 & 0.0 & 0 & -28.2 & -15 & 5 & 3.7 & 200 & -22.1 & -5 & 10 & 7.5 & 400 & -13.3 \\
\hline-25 & 0 & 0.0 & 100 & -18.9 & -15 & 5 & 3.7 & 300 & -17.2 & -5 & 15 & 11.2 & 0 & -41.3 \\
\hline-25 & 0 & 0.0 & 200 & -10.2 & -15 & 5 & 3.7 & 400 & -12.2 & -5 & 15 & 11.2 & 100 & -37.5 \\
\hline-25 & 0 & 0.0 & 300 & -2.0 & -15 & 10 & 7.5 & 0 & -44.6 & -5 & 15 & 11.2 & 200 & -33.7 \\
\hline-25 & 0 & 0.0 & 400 & 6.5 & -15 & 10 & 7.5 & 100 & -40.5 & -5 & 15 & 11.2 & 300 & -29.8 \\
\hline-25 & 5 & 3.7 & 0 & -44.5 & -15 & 10 & 7.5 & 200 & -36.3 & -5 & 15 & 11.2 & 400 & -25.0 \\
\hline-25 & 5 & 3.7 & 100 & -39.1 & -15 & 10 & 7.5 & 300 & -32.1 & 0 & 0 & 0.0 & 0 & -3.7 \\
\hline-25 & 5 & 3.7 & 200 & -33.8 & -15 & 10 & 7.5 & 400 & -27.6 & 0 & 0 & 0.0 & 100 & 4.9 \\
\hline-25 & 5 & 3.7 & 300 & -28.9 & -15 & 15 & 11.2 & 0 & -56.1 & 0 & 0 & 0.0 & 200 & 13.0 \\
\hline-25 & 5 & 3.7 & 400 & -23.7 & -15 & 15 & 11.2 & 100 & -52.3 & 0 & 0 & 0.0 & 300 & 20.9 \\
\hline-25 & 10 & 7.5 & 0 & -59.4 & -15 & 15 & 11.2 & 200 & -48.6 & 0 & 0 & 0.0 & 400 & 28.7 \\
\hline-25 & 10 & 7.5 & 100 & -54.6 & -15 & 15 & 11.2 & 300 & -44.7 & 0 & 5 & 3.7 & 0 & -13.9 \\
\hline-25 & 10 & 7.5 & 200 & -49.8 & -15 & 15 & 11.2 & 400 & -40.5 & 0 & 5 & 3.7 & 100 & -9.2 \\
\hline-25 & 10 & 7.5 & 300 & -45.5 & -10 & 0 & 0.0 & 0 & -13.5 & 0 & 5 & 3.7 & 200 & -4.1 \\
\hline-25 & 10 & 7.5 & 400 & -40.9 & -10 & 0 & 0.0 & 100 & -4.7 & 0 & 5 & 3.7 & 300 & 1.0 \\
\hline-25 & 15 & 11.2 & 0 & -72.8 & -10 & 0 & 0.0 & 200 & 3.7 & 0 & 5 & 3.7 & 400 & 6.2 \\
\hline-25 & 15 & 11.2 & 100 & -68.2 & -10 & 0 & 0.0 & 300 & 11.8 & 0 & 10 & 7.5 & 0 & -24.1 \\
\hline-25 & 15 & 11.2 & 200 & -63.5 & -10 & 0 & 0.0 & 400 & 19.9 & 0 & 10 & 7.5 & 100 & -20.0 \\
\hline-25 & 15 & 11.2 & 300 & -59.2 & -10 & 5 & 3.7 & 0 & -25.9 & 0 & 10 & 7.5 & 200 & -15.5 \\
\hline-25 & 15 & 11.2 & 400 & -55.1 & -10 & 5 & 3.7 & 100 & -21.1 & 0 & 10 & 7.5 & 300 & -10.7 \\
\hline-20 & 0 & 0.0 & 0 & -23.3 & -10 & 5 & 3.7 & 200 & -16.2 & 0 & 10 & 7.5 & 400 & -5.5 \\
\hline-20 & 0 & 0.0 & 100 & -14.2 & -10 & 5 & 3.7 & 300 & -11.4 & 0 & 15 & 11.2 & 0 & -33.7 \\
\hline-20 & 0 & 0.0 & 200 & -5.6 & -10 & 5 & 3.7 & 400 & -6.2 & 0 & 15 & 11.2 & 100 & -30.0 \\
\hline-20 & 0 & 0.0 & 300 & 2.5 & -10 & 10 & 7.5 & 0 & -37.8 & 0 & 15 & 11.2 & 200 & -25.6 \\
\hline-20 & 0 & 0.0 & 400 & 10.8 & -10 & 10 & 7.5 & 100 & -33.7 & 0 & 15 & 11.2 & 300 & -20.9 \\
\hline-20 & 5 & 3.7 & 0 & -38.1 & -10 & 10 & 7.5 & 200 & -29.5 & 0 & 15 & 11.2 & 400 & -15.6 \\
\hline-20 & 5 & 3.7 & 100 & -32.9 & -10 & 10 & 7.5 & 300 & -25.4 & 5 & 0 & 0.0 & 0 & 1.1 \\
\hline-20 & 5 & 3.7 & 200 & -28.0 & -10 & 10 & 7.5 & 400 & -20.9 & 5 & 0 & 0.0 & 100 & 9.7 \\
\hline-20 & 5 & 3.7 & 300 & -23.1 & -10 & 15 & 11.2 & 0 & -48.7 & 5 & 0 & 0.0 & 200 & 17.7 \\
\hline-20 & 5 & 3.7 & 400 & -18.0 & -10 & 15 & 11.2 & 100 & -45.0 & 5 & 0 & 0.0 & 300 & 25.5 \\
\hline-20 & 10 & 7.5 & 0 & -51.7 & -10 & 15 & 11.2 & 200 & -41.1 & 5 & 0 & 0.0 & 400 & 33.1 \\
\hline-20 & 10 & 7.5 & 100 & -47.1 & -10 & 15 & 11.2 & 300 & -37.3 & 5 & 5 & 3.7 & 0 & -7.8 \\
\hline-20 & 10 & 7.5 & 200 & -43.0 & -10 & 15 & 11.2 & 400 & -33.1 & 5 & 5 & 3.7 & 100 & -2.8 \\
\hline-20 & 10 & 7.5 & 300 & -38.8 & -5 & 0 & 0.0 & 0 & -8.6 & 5 & 5 & 3.7 & 200 & 2.2 \\
\hline-20 & 10 & 7.5 & 400 & -34.3 & -5 & 0 & 0.0 & 100 & 0.1 & 5 & 5 & 3.7 & 300 & 7.2 \\
\hline-20 & 15 & 11.2 & 0 & -64.0 & -5 & 0 & 0.0 & 200 & 8.4 & 5 & 5 & 3.7 & 400 & 12.4 \\
\hline-20 & 15 & 11.2 & 100 & -59.5 & -5 & 0 & 0.0 & 300 & 16.4 & 5 & 10 & 7.5 & 0 & -17.0 \\
\hline-20 & 15 & 11.2 & 200 & -55.8 & -5 & 0 & 0.0 & 400 & 24.3 & 5 & 10 & 7.5 & 100 & -12.3 \\
\hline-20 & 15 & 11.2 & 300 & -52.1 & -5 & 5 & 3.7 & 0 & -19.9 & 5 & 10 & 7.5 & 200 & -7.6 \\
\hline-20 & 15 & 11.2 & 400 & -47.9 & -5 & 5 & 3.7 & 100 & -15.1 & 5 & 10 & 7.5 & 300 & -2.9 \\
\hline-15 & 0 & 0.0 & 0 & -18.4 & -5 & 5 & 3.7 & 200 & -10.3 & 5 & 10 & 7.5 & 400 & 2.3 \\
\hline-15 & 0 & 0.0 & 100 & -9.4 & -5 & 5 & 3.7 & 300 & -5.3 & 5 & 15 & 11.2 & 0 & -25.7 \\
\hline-15 & 0 & 0.0 & 200 & -1.0 & -5 & 5 & 3.7 & 400 & 0.0 & 5 & 15 & 11.2 & 100 & -21.0 \\
\hline-15 & 0 & 0.0 & 300 & 7.3 & -5 & 10 & 7.5 & 0 & -31.0 & 5 & 15 & 11.2 & 200 & -16.2 \\
\hline-15 & 0 & 0.0 & 400 & 15.2 & -5 & 10 & 7.5 & 100 & -26.9 & 5 & 15 & 11.2 & 300 & -11.5 \\
\hline-15 & 5 & 3.7 & 0 & -31.9 & -5 & 10 & 7.5 & 200 & -22.8 & 5 & 15 & 11.2 & 400 & -6.3 \\
\hline-15 & 5 & 3.7 & 100 & -27.0 & -5 & 10 & 7.5 & 300 & -18.6 & & & & & \\
\hline
\end{tabular}


Table 2. Predicted standard operative temperatures $\left(T_{e f}\right)$ for full-fed, adult mule deer standing in snow-free open habitats in winter. $T_{a}$ is air temperature; $u$ is wind speed at $3 \mathrm{~m} ; u_{\mathrm{a}}$ is wind speed at animal height; $S W$ is short-wave radiation.

\begin{tabular}{|c|c|c|c|c|c|c|c|c|c|c|c|c|c|c|}
\hline $\begin{array}{l}T_{t} \\
\left({ }^{\circ} \mathrm{C}\right)\end{array}$ & $\underset{(\mathbf{m} / \mathbf{s})}{\mathbf{u}}$ & $\underset{(\mathbf{m} / \mathbf{s})}{\mathbf{u}_{\mathbf{s}}}(\mathrm{V}$ & $\underset{\left.\mathbf{W} / \mathbf{m}^{2}\right)}{S W}$ & $\begin{array}{r}T_{\infty} \\
\left({ }^{\circ} \mathrm{C}\right)\end{array}$ & $\begin{array}{r}T_{*} \\
\left({ }^{\circ} \mathrm{C}\right)\end{array}$ & $\underset{(\mathrm{m} / \mathrm{s})}{\mathrm{u}}$ & $\begin{array}{c}u_{k} \\
(m / s)\end{array}$ & $\underset{\left.W / \mathbf{m}^{2}\right)}{S W}$ & $\begin{array}{r}T_{\infty} \\
\left({ }^{\circ} \mathrm{C}\right)\end{array}$ & $\begin{array}{r}T_{a} \\
\left({ }^{\circ} \mathrm{C}\right)\end{array}$ & $\underset{(m / s)}{u}$ & $\underset{(\mathrm{m} / \mathrm{S})}{\mathbf{u}_{k}}($ & $\begin{array}{c}\mathbf{S W} \\
\left(\mathrm{W} / \mathrm{m}^{2}\right)\end{array}$ & $\begin{array}{r}T_{\infty} \\
\left({ }^{\circ} \mathrm{C}\right)\end{array}$ \\
\hline-25 & 0 & 0.0 & 0 & -28.2 & -15 & 5 & 3.9 & 200 & -25.5 & -5 & 10 & 7.7 & 400 & -19.8 \\
\hline-25 & 0 & 0.0 & 100 & -21.7 & -15 & 5 & 3.9 & 300 & -22.1 & -5 & is & 11.6 & 0 & -42.2 \\
\hline-25 & 0 & 0.0 & 200 & -15.5 & -15 & 5 & 3.9 & 400 & -18.3 & -5 & 15 & 11.6 & 100 & -39.5 \\
\hline-25 & 0 & 0.0 & 300 & -9.3 & -15 & 10 & 7.7 & 0 & -45.3 & -5 & 15 & 11.6 & 200 & -36.9 \\
\hline-25 & 0 & 0.0 & 400 & -3.0 & -15 & 10 & 7.7 & 100 & -42.4 & -5 & 15 & 11.6 & 300 & -34.2 \\
\hline-25 & 5 & 3.9 & 0 & -44.9 & -15 & 10 & 7.7 & 200 & -39.6 & -5 & 15 & 11.6 & 400 & -31.2 \\
\hline-25 & 5 & 3.9 & 100 & -41.2 & -15 & 10 & 7.7 & 300 & -36.7 & 0 & 0 & 0.0 & 0 & -3.7 \\
\hline-25 & 5 & 3.9 & 200 & -37.5 & -15 & 10 & 7.7 & 400 & -33.4 & 0 & 0 & 0.0 & 100 & 2.3 \\
\hline-25 & 5 & 3.9 & 300 & -33.9 & -15 & 15 & 11.6 & 0 & -57.1 & 0 & 0 & 0.0 & 200 & 8.4 \\
\hline-25 & 5 & 3.9 & 400 & $-\mathbf{3 0 . 0}$ & -15 & 15 & 11.6 & 100 & -54.5 & 0 & 0 & 0.0 & 300 & 13.9 \\
\hline-25 & 10 & 7.7 & 0 & -60.2 & -15 & 15 & 11.6 & 200 & -51.9 & 0 & 0 & 0.0 & 400 & 19.7 \\
\hline-25 & 10 & 7.7 & 100 & -56.9 & -15 & 15 & 11.6 & 300 & -49.2 & 0 & 5 & 3.9 & 0 & -14.2 \\
\hline-25 & 10 & 7.7 & 200 & 53.5 & -15 & 15 & 11.6 & 400 & -46.2 & 0 & 5 & 3.9 & 100 & -10.9 \\
\hline-25 & 10 & 7.7 & 300 & -50.2 & -10 & 0 & 0.0 & 0 & -13.5 & 0 & 5 & 3.9 & 200 & -7.5 \\
\hline-25 & 10 & 7.7 & 400 & -46.8 & -10 & 0 & 0.0 & 100 & -7.3 & 0 & 5 & 3.9 & 300 & -4.0 \\
\hline-25 & 15 & 11.6 & 0 & -73.9 & -10 & 0 & 0.0 & 200 & -1.1 & 0 & 5 & 3.9 & 400 & 0.0 \\
\hline-25 & 15 & 11.6 & 100 & -70.7 & -10 & 0 & 0.0 & 300 & 4.6 & 0 & 10 & 7.7 & 0 & -24.7 \\
\hline-25 & 15 & 11.6 & 200 & -67.5 & -10 & 0 & 0.0 & 400 & 10.6 & 0 & 10 & 7.7 & 100 & -21.9 \\
\hline-25 & 15 & 11.6 & 300 & -64.3 & -10 & 5 & 3.9 & 0 & -26.2 & 0 & 10 & 7.7 & 200 & -19.0 \\
\hline-25 & 15 & 11.6 & 400 & -60.8 & -10 & 5 & 3.9 & 100 & -22.9 & 0 & 10 & 7.7 & 300 & -15.6 \\
\hline-20 & 0 & 0.0 & 0 & -23.3 & -10 & 5 & 3.9 & 200 & -19.5 & 0 & 10 & 7.7 & 400 & -11.9 \\
\hline-20 & 0 & 0.0 & 100 & -17.0 & -10 & 5 & 3.9 & 300 & -16.2 & 0 & 15 & 11.6 & 0 & -34.6 \\
\hline-20 & 0 & 0.0 & 200 & -10.8 & -10 & 5 & 3.9 & 400 & -12.4 & 0 & 15 & 11.6 & 100 & -32.0 \\
\hline-20 & 0 & 0.0 & 300 & -4.7 & -10 & 10 & 7.7 & 0 & -38.5 & 0 & 15 & 11.6 & 200 & -29.3 \\
\hline-20 & 0 & 0.0 & 400 & 1.5 & -10 & 10 & 7.7 & 100 & -35.6 & 0 & 15 & 116 & 300 & -26.1 \\
\hline-20 & 5 & 3.9 & 0 & -38.5 & -10 & 10 & 7.7 & 200 & -32.8 & 0 & 15 & 11.6 & 400 & -22.3 \\
\hline-20 & 5 & 3.9 & 100 & -34.8 & -10 & 10 & 7.7 & 300 & -29.9 & 5 & 0 & 0.0 & 0 & 1.1 \\
\hline-20 & 5 & 3.9 & 200 & -31.4 & -10 & 10 & 7.7 & 400 & -26.6 & 5 & 0 & 0.0 & 100 & 7.1 \\
\hline-20 & 5 & 3.9 & 300 & -28.0 & -10 & 15 & 11.6 & 0 & -49.7 & 5 & 0 & 0.0 & 200 & 13.1 \\
\hline-20 & 5 & 3.9 & 400 & -24.1 & .10 & 15 & 11.6 & 100 & -47.1 & 5 & 0 & 0.0 & 300 & 18.6 \\
\hline-20 & 10 & 7.7 & 0 & -52.5 & -10 & 15 & 11.6 & 200 & -44.4 & 5 & 0 & 0.0 & 400 & 24.5 \\
\hline-20 & 10 & 7.7 & 100 & -49.1 & -10 & 15 & 11.6 & 300 & -41.8 & 5 & 5 & 3.9 & 0 & -8.0 \\
\hline-20 & 10 & 7.7 & 200 & -46.3 & -10 & 15 & 11.6 & 400 & -38.8 & 5 & 5 & 3.9 & 100 & -4.6 \\
\hline-20 & 10 & 7.7 & 300 & -43.4 & -5 & 0 & 0.0 & 0 & -8.6 & 5 & 5 & 3.9 & 200 & -1.1 \\
\hline-20 & 10 & 7.7 & 400 & -40.2 & -5 & 0 & 0.0 & 100 & -2.5 & 5 & 5 & 3.9 & 300 & 2.4 \\
\hline-20 & 15 & 11.6 & 0 & -65.0 & -5 & 0 & 0.0 & 200 & 3.6 & 5 & 5 & 3.9 & 400 & $\begin{array}{l}2.4 \\
6.4\end{array}$ \\
\hline-20 & 15 & 11.6 & 100 & -61.8 & -5 & 0 & 0.0 & 300 & 9.2 & 5 & 10 & 7.7 & 0 & -17.5 \\
\hline-20 & 15 & 11.6 & 200 & -59.2 & -5 & 0 & 0.0 & 400 & 15.1 & 5 & 10 & 7.7 & 100 & -14.2 \\
\hline-20 & 15 & 11.6 & 300 & -56.6 & -5 & 5 & 3.9 & 0 & -20.2 & 5 & 10 & 77 & 200 & -10.9 \\
\hline-20 & 15 & 11.6 & 400 & -53.6 & -5 & 5 & 3.9 & 100 & -16.9 & 5 & 10 & 7.7 & 300 & -7.6 \\
\hline-15 & 0 & 0.0 & 0 & -18.4 & -5 & 5 & 3.9 & 200 & -13.6 & 5 & 10 & 7.7 & 400 & -3.9 \\
\hline-15 & 0 & 0.0 & 100 & -12.1 & -5 & 5 & 3.9 & 300 & -10.3 & 5 & 15 & 11.6 & 0 & -26.5 \\
\hline-15 & 0 & 0.0 & 200 & -6.1 & -5 & 5 & 3.9 & 400 & -6.3 & 5 & 15 & 11.6 & 100 & -23.1 \\
\hline-15 & 0 & 0.0 & 300 & -0.1 & -5 & 10 & 7.7 & 0 & -31.6 & 5 & 15 & 11.6 & 200 & -19.8 \\
\hline-15 & 0 & 0.0 & 400 & 6.0 & -5 & 10 & 7.7 & 100 & -28.8 & 5 & 15 & 11.6 & 300 & -16.5 \\
\hline-15 & 5 & 3.9 & 0 & -32.2 & -5 & 10 & 7.7 & 200 & -25.9 & 5 & 15 & 11.6 & 400 & -12.7 \\
\hline-15 & 5 & 3.9 & 100 & -28.9 & -5 & 10 & 7.7 & 300 & -23.0 & & & & & \\
\hline
\end{tabular}

$\mathrm{C}$ from nighttime conditions. Under the same environmental conditions in both seasons (e.g., $T_{\mathrm{a}}=0^{\circ} \mathrm{C}, \mathrm{u}=0 \mathrm{~m} / \mathrm{s}, \mathrm{SW}=400 \mathrm{~W} / \mathrm{m}^{2}$ ), the standard operative temperature experienced by the animal is greater in winter because of the greater insulation and absorptivity to solar radiation provided by its winter pelage.

\section{Lower and Upper Critical Temperatures}

Environmental conditions between the predicted surfaces of lower (LCT) and upper critical temperatures (UCT) represent optimum thermal environments for mule deer (Figs. 1-3). Increased energy expenditures for thermoregulation would occur above and below these surfaces. Variations in the steepness of the surfaces represent the sensitivity of the boundary between an animal's critical and noncritical thermal environment to different weather variables. In winter, the range of air temperatures in which animals exist most comfortably (i.e., the width of the thermoneutral zone) under conditions of minimal wind is $\sim 22^{\circ} \mathrm{C}$ with no solar gain and $24-25^{\circ} \mathrm{C}$ with full solar loads ( $400 \mathrm{~W} / \mathrm{m}^{2}$; Figs. 1-2). Increasing wind speeds to $15 \mathrm{~m} / \mathrm{s}$ decreases the thermoneutral zone to $\sim 11^{\circ} \mathrm{C}$, regardless of incident solar levels. In summer, high wind speeds ( 15 $\mathrm{m} / \mathrm{s}$ ) reduce the thermoneutral zone by $8-10^{\circ} \mathrm{C}$ from still air conditions (Fig. 3).

\section{Discussion}

\section{Implications of Predicted Thermal Environments}

Predicted thermal environments for mule deer provide insight into the contributions of solar radiation and wind to the standard operative temperatures experienced by animals, seasonal differences in these contributions, the importance of snow relative to solar reflectivity and the wind speed profile, and the importance of reduced radiative heat input from a clear sky at night.

Particular importance of solar radiation to mule deer at cold temperatures on snow-covered surfaces during winter is demonstrated by animals that are not thermally stressed until $T_{a}$ 's fall to approximately $-53^{\circ} \mathrm{C}$ when solar radiation levels are high (400 
Table 3. Predicted standard operative temperatures $\left(T_{e}\right)$ for full-fed, adult mule deer standing in open habitats in summer. $T_{a}$ is air temperature; $u$ in wind speed at $3 \mathrm{~m} ; u_{\mathrm{a}}$ is wind speed at animal height; SW is short-wave radiation.

\begin{tabular}{|c|c|c|c|c|c|c|c|c|c|c|c|c|c|c|}
\hline $\begin{array}{l}T_{a} \\
\left({ }^{\circ} \mathrm{C}\right)\end{array}$ & $\underset{(\mathbf{m} / \mathbf{s})}{\mathbf{u}}$ & $\begin{array}{r}u_{u_{n}} \\
(m / s)\end{array}$ & $\underset{\left(\mathbf{W} / \mathbf{m}^{2}\right)}{\mathbf{S W}}$ & $\begin{array}{r}T_{E} \\
\left({ }^{\circ} \mathrm{C}\right)\end{array}$ & $\begin{array}{r}T_{a} \\
\left({ }^{\circ} \mathrm{C}\right)\end{array}$ & $\underset{(m / s)}{u}$ & $\underset{(m / s)}{u_{2}}$ & $\begin{array}{c}\mathbf{S W} \\
\left(\mathbf{W} / \mathbf{m}^{2}\right)\end{array}$ & $\begin{array}{r}T_{\infty} \\
\left({ }^{\circ} \mathrm{C}\right)\end{array}$ & $\begin{array}{r}T_{2} \\
\left({ }^{\circ} \mathrm{C}\right)\end{array}$ & $\underset{(\mathrm{m} / \mathrm{s})}{\mathbf{u}}$ & $\underset{(m / S)}{u_{a}}$ & $\begin{array}{c}\mathbf{S W} \\
\left(\mathbf{W} / \mathbf{m}^{2}\right)\end{array}$ & $\begin{array}{r}T_{\infty} \\
\left({ }^{\circ} \mathrm{C}\right)\end{array}$ \\
\hline 0 & 0 & 0.0 & 0 & -3.7 & 10 & 5 & 3.7 & 400 & 10.9 & 20 & 10 & 7.5 & 800 & 24.4 \\
\hline 0 & 0 & 0.0 & 200 & 6.5 & 10 & 5 & 3.7 & 600 & 16.2 & 20 & 15 & 11.2 & 0 & 5.0 \\
\hline 0 & 0 & 0.0 & 400 & 16.0 & 10 & 5 & 3.7 & 800 & 17.7 & 20 & 15 & 11.2 & 200 & 9.0 \\
\hline 0 & 0 & 0.0 & 600 & 24.9 & 10 & 10 & 7.5 & 0 & -4.5 & 20 & 15 & 11.2 & 400 & 13.4 \\
\hline 0 & 0 & 0.0 & 800 & 27.5 & 10 & 10 & 7.5 & 200 & -0.2 & 20 & 15 & 11.2 & 600 & 18.7 \\
\hline 0 & 5 & 3.7 & 0 & -11.7 & 10 & 10 & 7.5 & 400 & 4.1 & 20 & 15 & 11.2 & 800 & 20.2 \\
\hline 0 & 5 & 3.7 & 200 & -5.9 & 10 & 10 & 7.5 & 600 & 8.6 & 25 & 0 & 0.0 & 0 & 20.7 \\
\hline 0 & 5 & 3.7 & 400 & -0.4 & 10 & 10 & 7.5 & 800 & 9.9 & 25 & 0 & 0.0 & 200 & 30.2 \\
\hline 0 & 5 & 3.7 & 600 & 4.8 & 10 & 15 & 11.2 & 0 & -8.8 & 25 & 0 & 0.0 & 400 & 39.1 \\
\hline 0 & 5 & 3.7 & 800 & 6.1 & 10 & 15 & 11.2 & 200 & -4.9 & 25 & 0 & 0.0 & 600 & 47.1 \\
\hline 0 & 10 & 7.5 & 0 & -18.5 & 10 & 15 & 11.2 & 400 & -1.0 & 25 & 0 & 0.0 & 800 & 49.4 \\
\hline 0 & 10 & 7.5 & 200 & -13.4 & 10 & 15 & 11.2 & 600 & 3.0 & 25 & 5 & 3.7 & 0 & 18.1 \\
\hline 0 & 10 & 7.5 & 400 & -8.4 & 10 & 15 & 11.2 & 800 & 4.2 & 25 & 5 & 3.7 & 200 & 23.6 \\
\hline 0 & 10 & 7.5 & 600 & -4.1 & 15 & 0 & 0.0 & 0 & 11.0 & 25 & 5 & 3.7 & 400 & 29.1 \\
\hline 0 & 10 & 7.5 & 800 & -2.8 & 15 & 0 & 0.0 & 200 & 20.7 & 25 & 5 & 3.7 & 600 & 34.4 \\
\hline 0 & 15 & 11.2 & 0 & -24.0 & 15 & 0 & 0.0 & 400 & 29.7 & 25 & 5 & 3.7 & 800 & 36.1 \\
\hline 0 & 15 & 11.2 & 200 & -19.2 & 15 & 0 & 0.0 & 600 & 38.4 & 25 & 10 & 7.5 & 0 & 14.9 \\
\hline 0 & 15 & 11.2 & 400 & -14.3 & 15 & 0 & 0.0 & 800 & 40.5 & 25 & 10 & 7.5 & 200 & 20.1 \\
\hline 0 & 15 & 11.2 & 600 & -10.4 & 15 & 5 & 3.7 & 0 & 6.4 & 25 & 10 & 7.5 & 400 & 25.3 \\
\hline 0 & 15 & 11.2 & 800 & -9.3 & 15 & 5 & 3.7 & 200 & 11.5 & 25 & 10 & 7.5 & 600 & 30.5 \\
\hline 5 & 0 & 0.0 & 0 & 1.2 & 15 & 5 & 3.7 & 400 & 16.7 & 25 & 10 & 7.5 & 800 & 32.0 \\
\hline 5 & 0 & 0.0 & 200 & 11.2 & 15 & 5 & 3.7 & 600 & 22.2 & 25 & 15 & 11.2 & 0 & 12.1 \\
\hline 5 & 0 & 0.0 & 400 & 20.5 & 15 & 5 & 3.7 & 800 & 23.9 & 25 & 15 & 11.2 & 200 & 17.4 \\
\hline 5 & 0 & 0.0 & 600 & 29.3 & 15 & 10 & 7.5 & 0 & 1.8 & 25 & 15 & 11.2 & 400 & 22.6 \\
\hline 5 & 0 & 0.0 & 800 & 31.9 & 15 & 10 & 7.5 & 200 & 6.2 & 25 & 15 & 11.2 & 600 & 27.7 \\
\hline 5 & 5 & 3.7 & 0 & -5.3 & 15 & 10 & 7.5 & 400 & 10.6 & 25 & 15 & 11.2 & 800 & 29.3 \\
\hline 5 & 5 & 3.7 & 200 & 0.0 & 15 & $10^{\circ}$ & 7.5 & 600 & 15.1 & 30 & 0 & 0.0 & 0 & 25.5 \\
\hline 5 & 5 & 3.7 & 400 & 5.2 & 15 & 10 & 7.5 & 800 & 16.7 & 30 & 0 & 0.0 & 200 & 34.9 \\
\hline 5 & 5 & 3.7 & 600 & 10.5 & 15 & 15 & 11.2 & 0 & -2.0 & 30 & 0 & 0.0 & 400 & 43.7 \\
\hline 5 & 5 & 3.7 & 800 & 12.1 & 15 & 15 & 11.2 & 200 & 1.9 & 30 & 0 & 0.0 & 600 & 51.6 \\
\hline 5 & 10 & 7.5 & 0 & -11.1 & 15 & 15 & 11.2 & 400 & 6.0 & 30 & 0 & 0.0 & 800 & 53.8 \\
\hline 5 & 10 & 7.5 & 200 & -6.5 & 15 & 15 & 11.2 & 600 & 10.0 & 30 & 5 & 3.7 & 0 & 24.5 \\
\hline 5 & 10 & 7.5 & 400 & -2.2 & 15 & 15 & 11.2 & 800 & 11.2 & 30 & 5 & 3.7 & 200 & 29.9 \\
\hline 5 & 10 & 7.5 & 600 & 2.2 & 20 & 0 & 0.0 & 0 & 15.8 & 30 & 5 & 3.7 & 400 & 35.2 \\
\hline 5 & 10 & 7.5 & 800 & 3.5 & 20 & 0 & 0.0 & 200 & 25.4 & 30 & 5 & 3.7 & 600 & 40.5 \\
\hline 5 & 15 & 11.2 & 0 & -15.7 & 20 & 0 & 0.0 & 400 & 34.6 & 30 & 5 & 3.7 & 800 & 42.3 \\
\hline 5 & 15 & 11.2 & 200 & -11.5 & 20 & 0 & 0.0 & 600 & 42.8 & 30 & 10 & 7.5 & 0 & 22.8 \\
\hline 5 & 15 & 11.2 & 400 & -7.7 & 20 & 0 & 0.0 & 800 & 45.1 & 30 & 10 & 7.5 & 200 & 27.9 \\
\hline 5 & 15 & 11.2 & 600 & $-\mathbf{3 . 8}$ & 20 & 5 & 3.7 & 0 & 12.3 & 30 & 10 & 7.5 & 400 & 33.0 \\
\hline 5 & 15 & 11.2 & 800 & -2.6 & 20 & 5 & 3.7 & 200 & 17.3 & 30 & 10 & 7.5 & 600 & 37.7 \\
\hline 10 & 0 & 0.0 & 0 & 6.1 & 20 & 5 & 3.7 & 400 & 22.8 & 30 & 10 & 7.5 & 800 & 39.1 \\
\hline 10 & 0 & 0.0 & 200 & 16.0 & 20 & 5 & 3.7 & 600 & 28.4 & 30 & 15 & 11.2 & 0 & 21.4 \\
\hline 10 & 0 & 0.0 & 400 & 25.1 & 20 & 5 & 3.7 & 800 & 30.0 & 30 & 15 & 11.2 & 200 & 26.6 \\
\hline 10 & 0 & 0.0 & 600 & 34.0 & 20 & 10 & 7.5 & 0 & 8.3 & 30 & 15 & 11.2 & 400 & 31.7 \\
\hline 10 & 0 & 0.0 & 800 & 36.3 & 20 & 10 & 7.5 & 200 & 12.7 & 30 & 15 & 11.2 & 600 & 36.3 \\
\hline 10 & 5 & 3.7 & 0 & 0.6 & 20 & 10 & 7.5 & 400 & 17.5 & 30 & 15 & 11.2 & 800 & 37.4 \\
\hline 10 & 5 & 3.7 & 200 & 5.7 & 20 & 10 & 7.5 & 600 & 22.8 & & & & & \\
\hline
\end{tabular}

$\left.W / m^{2}\right)$ and effects of wind are minimal (Fig. 1). Even at $T_{a}$ 's of $-30^{\circ} \mathrm{C}$, the first direct beam radiation at sunrise will stop shivering by mule deer almost immediately and elevate skin temperatures equal to those at $+30^{\circ} \mathrm{C}$ in summer (Parker and Robbins 1984). Radiant temperatures of the surface of mule deer pelage may change $18^{\circ} \mathrm{C}$ between shaded and unshaded conditions (Parker and Harlan 1972). In contrast, upper critical temperatures actually may be exceeded at $-25^{\circ} \mathrm{C}\left(T_{n}\right)$ for animals on snow surfaces with minimal wind speeds and high solar levels $\left(400 \mathrm{~W} / \mathrm{m}^{2}\right)$ (Fig. 1, Table 1).

In high elevation mountain habitats when air temperatures fall to freezing $\left(0^{\circ} \mathrm{C}\right)$ in late summer, solar levels must exceed 175 $\mathrm{W} / \mathrm{m}^{2}$ for adults to remain in thermoneutral environments (Fig. 3). As such, nighttime poses lower thermally critical conditions in still air. In the extreme case, when freezing air temperatures occur at midday, solar radiation levels greater than $550 \mathrm{~W} / \mathrm{m}^{2}$ may induce heat stress. Hence, a singular change in solar radiation from min- imal to average daily loads will cause standard operative temperatures for mule deer to range from cold-stressed to heat-stressed environments.

In both seasons, under conditions of dark, calm, and no wind, an animal radiates more heat to the environment than it gains. It consequently senses the environment to be colder than air temperature (e.g., Table 1, adult in snow in winter: $T_{\mathrm{a}}=-20^{\circ} \mathrm{C}, \mathrm{u}=0, \mathrm{SW}=$ $0, T_{0=}=-23.3^{\circ} \mathrm{C}$ ). In the model, this occurs because the long-wave radiation absorbed by the animal is less than its long-wave emittance. Data for white-tailed deer (Odocoileus virginianus) suggest that animal surface temperatures $\left(T_{r}\right)$ are greater than air temperatures under still, no wind conditions $\left(T_{\mathrm{a}}=0.0\right.$ to $-30.0^{\circ} \mathrm{C}, T_{\mathrm{r}}=6.6$ to $-21.8^{\circ} \mathrm{C}$; Moen 1968b). Because this model assumed an outer coat temperature equal to air temperature, net radiative heat loss and an estimate of the resistance of free convection by mule deer may be slightly greater than predicted here and their operative thermal environment would be even colder. 
Wind also significantly affects the mule deer's thermal environment. The range of temperatures within the thermoneutral zone under no wind conditions in winter declines $50 \%$ when wind speeds reach $15 \mathrm{~m} / \mathrm{s} ;$ similarly, in summer a $43 \%$ decrease occurs. Increasing wind speeds ( 0 to $15 \mathrm{~m} / \mathrm{s})$ have the greatest effect on lower critical environments with high solar radiation levels; effects are least on upper thermally critical limits during no sun conditions (Figs. 1-3).

The wind speed experienced by an animal is slightly less when it stands in snow (or a meadow) that is $0.1 \mathrm{~m}$ deep than when on bare ground because the wind speed profile, which increases exponentially with increasing height above the ground (Campbell 1977:39), does not begin until $0.1 \mathrm{~m}$ above the substrate. Standard operative temperatures experienced by fawns would be slightly colder than those experienced by adults (Tables 1-3). Even though smaller animals (with lower rib-cage heights) have the advantage of being in the lower-velocity portion of a wind speed profile (Moen and Jacobsen 1975), their characteristic dimension relative to body weight (Parker 1987) and larger surface area to volume ratio result in higher susceptibility to convective heat losses.

\section{Limitations of Estimating Thermally Critical Environments}

Critical temperatures for mule deer were determined for standing, full-fed, healthy animals. Thermal stress for animals in the field may be adjusted slightly by behavior, diet, and body condition. For example, lying postures potentially reduce heat loss through the extremities and may decrease LCT's for moose (Alces alces) by more than $10^{\circ} \mathrm{C}$ (Renecker et al. 1978). In contrast, locomotory costs in reindeer may substitute for some thermoregulatory expenditures (Nilssen et al. 1984), even though convective heat losses would be greater for moving than for stationary animals. Fasting pronghorn (Antilocapra americana) reach critical thermal environments at air temperatures $12-15^{\circ} \mathrm{C}$ higher than those on ad libitum intake (Wesley et al. 1973). For free-ranging mule deer in poor body condition or on limited food intake in winter, lying postures would minimize energy expenditures for activity, and, because of the insulative qualities of snow, reduce thermoregulatory costs. A reduced food intake or quality-limited diet, however, also would reduce heat produced by the animal. Therefore, lower critical temperatures for free-ranging mule deer would probably approach $-19^{\circ} \mathrm{C}$, as used in this model, and also determined by Mautz et al. (1985), by accumulative effects of food and posture. Regardiess of the absolute value chosen to represent the boundary between thermoneutrality and thermally critical environments, the relative effects of air temperature, wind speed, and solar radiation should be as shown in the surfaces generated by our analysis.

Weight-specific metabolic rates of fawns may be significantly higher than those of adults depending on age of the animal (Parker and Robbins 1984). As such, feed intake per unit weight must be higher for fawns. Young animals would be affected indirectly to a greater extent by thermal constraints than would adults.

Convective heat losses, as estimated in this model, are based on a static nature of the animal's hair coat. For animals in winter, wind speeds up to $15 \mathrm{~m} / \mathrm{s}$ reduce coat resistance to $\approx 35 \%$ of still-air values, which is only slightly more than the insulation determined for deer pelts by Moote (1955). Winter pelage of deer provides poor insulation from wind, particularly in comparison to arctic species, because it is less dense and easily compressed. At some undetermined wind speed, however, this maximum protective insulation of the hair coat, regardless of its relative quality, will be disrupted. Studies using animal models with sheep and cattle pelts indicate that external insulation is partially destroyed because of a breakdown and/or separation of the hair fibers at wind speeds greater than $11 \mathrm{~m} / \mathrm{s}$ (Ames and Insley 1975). If these data are applicable to live animals, coat resistance would be reduced greatly from that predicted by this model and standard operative temperatures at wind speeds greater than $11 \mathrm{~m} / \mathrm{s}$ would be much colder. Unfortuntely, actual micrometeorological measurements of high wind speeds have not been related to metabolic requirements of mule deer.

Conductive heat losses have not been included in this model. In snow-covered habitats in winter, the insulation provided by the animal's winter pelage and the snow may minimize heat losses. For animals lying on cold, poorly insulated ground without snow or during early spring and summer conditions, conduction may aggravate cold stress and may be one of the more important ways of heat loss (Gatenby 1977). Standard operative environments would be colder than predicted herein if the heat lost by conduction (but conserved by lying) exceeded heat lost by convection (and expended during standing).

The transitions between different thermal environments (the animal's transient state) is a more difficult variable to incorporate in thermal models (Campbell 1977:96, Parker and Robbins 1985). Animals are not static components of their environment. Because of activity patterns and movements, a variety of non-uniform microsites are encountered. Large animals, with low surface area to volume ratios and a large thermal resistance, are able to endure long exposures to environments outside acceptable operative conditions before significant changes in body temperature occur. Smaller animals respond more quickly to environmental changes and consequently may benefit to a greater degree, at least in the short term, by the provision of thermal cover.

\section{Influences of Vegetative Cover}

We have modelled the interactions of weather variables as they affect the operative temperatures experienced by adult mule deer. The animal's metabolic response to a given $T_{\infty}$ is the same no matter what habitat it is in. It is unlikely, however, that abiotic variables measured in open areas are equivalent to those measured simultaneously under forested canopies. Vegetative structures (and hence, the concept of thermal cover) modify the abiotic components of open areas (temperature, windspeed, radiation) and have been addressed by Bunnell et al. (1986).

From a very general standpoint, compared with an open area, a forested habitat is often cooler $\left(-2^{\circ} \mathrm{C}\right)$ in summer and warmer $\left(+2^{\circ}\right.$ C) in winter, may reduce windspeeds by $85 \%$, receives only $20 \%$ of total incoming short-wave radiation, and traps more long-wave radiation at night (Bunnell et al. 1986). Recognizing that there are additional complexities (e.g., changes in albedo, snow depths, and turbulence factors), but simplistically assuming only the above differences between open and forested areas, we compared estimates of $T_{w}$ between the 2 areas during winter and summer. $T_{\omega}$ was significantly altered in these simulations. For example at $-10^{\circ} \mathrm{C}$ with windspeeds of $10 \mathrm{~m} / \mathrm{s}$ under snow conditions during winter, mule deer are thermally stressed regardless of solar radiation level (Table 1). $T_{\omega}$ in forested habitats would be $15^{\circ} \mathrm{C}$ warmer than in open habitats because of reduced windspeeds, thereby eliminating thermal stress. Similarly in summer, under calm conditions and $20^{\circ} \mathrm{C}$, forested areas may provide shaded areas that are $20^{\circ} \mathrm{C}$ cooler than open areas by reducing high solar levels. Shrub-steppe communities will have less profound global effects on altering weather variables, but scattered trees or shrubs, rocks, or topological formations may, nonetheless, provide improved microclimates for deer.

Further studies are needed to compare concurrent measurements of micrometeorological variables in open and forested areas; modelling efforts to predict the environmental conditions imposed by vegetative structures from weather measurements in open areas would be extremely valuable and could then be linked with this model to predict whether or not an animal is thermally stressed. 


\section{Conclusions}

Management of mule deer and their habitats should integrate the behavioral, physiological, and microclimatic factors that cause changes in habitat use over the year. Maintaining a healthy, productive deer population depends on how well and how often the physiological needs of the individuals are met or exceeded (Leckenby 1977). This model has addressed only one component of the individual's energy balance-thermoregulation. Effective management also must consider the relative contributions of activity, diet, and sociality to energy requirements and subsequent population status.

An animal's habitat choice may not directly reflect thermal conditions (see Bunnell et al. 1986:17-24). During summer, the thermoregulatory cost of remaining in the open may be counterbalanced by opportunities for increased energy intake from abundant and high quality forage. In winter, however, at a time of decreased forage resources and increased locomotory costs in snow, it is less likely that animals will leave thermal cover on the comparatively rarer chance of finding sufficient energy intake to meet additional thermoregulatory requirements. Thermal cover serves to reduce heat loss or gain and becomes physiologically important when its presence is necessary to maintain a positive energy balance (Moen 1968b). Daily thermoregulatory costs during winter are often of longer duration than periodic locomotory costs and can be a constant drain on an individual's reserves. The energy expended during thermal stress in summer may reduce what is available for lactation, growth, fattening, and movement (Leckenby 1977). Thermal stress in both seasons, therefore, may influence immediate survival of the individual, and production and future survival of its offspring.

Management guidelines for the provision of thermally neutral habitats for mule deer should delineate the range of standard operative temperatures that occur in habitats occupied by deer. Specifically measured micrometeorological variables are necessary for a better understanding of habitat influences on weather conditions and for the prediction of standard operative temperatures. The calculations of $T_{\omega}$ should incorporate region-specific geographical coordinates, average snow depths and vegetation heights, and average size of animals. Physiological responses of the animal to $T_{\boldsymbol{w}}$ are incorporated in this model to determine if animals are thermally stressed. Two questions should then be asked: (1) how often do animals encounter thermally critical environments during each season? (or to what degree could thermoregulation influence habitat selection?), and (2) if critical environments are often encountered, is thermal cover available to ameliorate existing thermal conditions or are food and water resources adequate to offset heat losses and gains? (or should silvicultural or management prescriptions be used to generate suitable thermal cover, food, or water resources?). Habitat management for thermal cover would be helpful under those conditions in which individuals are without large energy reserves and for which added energetic constraints would negatively influence population status.

\section{Literature Cited}

Ames, D.R., and L.W. Inaley. 1975. Wind-chill effect for cattle and sheep. J. Anim. Sci. 40:161-165.

Bakken, G.S. 1980. The use of standard operative temperature in the study of the thermal energetics of birds. Physiol. Zool. 53:108-119.

Bakken, G.S. 1981. How many equivalent black-body temperatures are there? J. Therm. Biol. 6:59-60.

Beall, R.C. 1974. Winter habitat selection and use by a western Montana elk herd. PhD. Thesis, Univ. Montana, Missoula.

Black, H., R.J. Scherdinger, and J.W. Thomas. 1976. Relationships of Rocky Mountain elk and Rocky Mountain mule deer habitat to timber management in the Blue Mountains of Oregon and Washington. p. 1 1-31. In: S.R. Hieb (ed), Proc. Elk-logging-roads Symp., Univ. Idaho. unnell, F.L., K.L. Parker, L.L. Krementer, and F.W. Hovey. 1986. Thermoregulation and thermal cover of deer and elk on Vancouver Island: problem analysis. B.C. Ministries of Environment and Forests. IWIFR28. Victoria, B.C.

Bundinger, J.A.1975. Aerodynamics of vegetated surfaces. In:D.A. deVries and N.H. Afgan (eds.), Heat and mass transfer in the biosphere. J. Wiley and Sons, New York.

Campbell, G.S. 1977. An introduction to environmental biophysics. Springer-Verlag, New York, N.Y.

Campbell, G.S. 1981. Fundamentals of radiation and temperature relations. p. 11-40 In: O.L. Lange, P.S. Nobel, C.B. Osmond, and H. Ziegler (eds), Encyclopedia of plant pathology, Vol. 12A, Physiological plant ecology I. Springer-Verlag, Berlin.

Campbell, G.S., A.J. McArthur, and J.L. Monteith. 1980. Windspeed dependence of heat and mass transfer through coats and clothing. Boundary-Layer Meterology 18:485-493.

Dixon, W.J. 1981. BMDP Statistical Software. Univ. California Press. Berkeley.

Gatenby, R.M. 1977. Conduction of heat from sheep to ground. Agr. Meteorol. 18:387-400.

ISSCO Visual information system software. 1987. DISSPLA: Display integrated software system and plotting language. Integrated Software Systems Corp. San Diego, Calif.

Leckenby, D.A. 1977. Management of mule deer and their habitats: applying concepts of behavior, physiology, and microclimate. West. Proc. Annu. Conf. West. Assoc. State Game and Fish Comm. 57:206-217.

Lowry, W.P. 1969. Weather and life; an introduction to biometerology. Academic Press, New York, N.Y.

Mautz, W.W., Pekins, P.J., and J.A. Warren. 1985. Cold temperature effects on metabolic rate of white-tailed, mule, and black-tailed deer in winter coat. p. 453-457. In: P.F. Fennessy and K.R. Drew (eds), Biology of deer production. R. Soc. N.Z. Bull. 22.

Moen, A.N. 1968a. Critical thermal environment: a new look at an old concept. Bioscience 18:1041-1043.

Moen, A.N. 1968b. Surface temperatures and radiant heat loss from whitetailed deer. J. Wildl. Manage. 32:338-344.

Moen, A.N. 1973. Wildlife ecology; an analytical approach. W.H. Freeman and Co., San Francisco, Calif.

Moen, A.N. 1974. Radiant temperatures of hair surfaces. J. Range Manage. 27:401-403.

Moen, A.N., and F.L. Jacobeen. 1974. Changes in radiant temperature of animal surfaces with wind and radiation. J. Wildl. Manage. 38:366-368.

Moen, A.N., and N.K. Jacobeen. 1975. Thermal exchange, physiology, and behavior of white-tailed deer. p. 509-524. In: D.M. Gates and R.B. Schmerl (eds), Perspectives of biophysical ecology. Springer-Verlag, New York, N.Y.

Monteth, J.L. 1973. Principles of environmental physics. American Elsevier, New York.

Moote, I. 1955. The thermal insulation of caribou pelts. Text. Res. J. 25:832-837.

Nilosen, K.J., H.K. Johmen, A. Rognmo, and A.S. BHix. 1984. Heart rate and energy expenditure in resting and running Svalbard and Norwegian reindeer. Amer. J. Physiol. 246 (Reg. Int. Comp. Physiol. 15): R963-R967.

Paltridge, G.W., and C.M.R. Platt. 1976. Radiative processes in meteorology and climatology. Elsevier Sci. Publ. Co., New York, N.Y.

Parker, H.D., Jr., and J.C. Harlan. 1972. Solar radiation affects radiant temperatures of a deer surface. USDA Forest Serv. Res. Note RM-215.

Parker, K.L. 1983. Ecological energetics of mule deer and elk: locomotion and thermoregulation. Ph.D. Thesis, Washington State Univ., Pullman.

Parker, K.L. 1987. Body-surface measurements of mule deer and elk. J. Wildl. Manage. 51:630-633.

Parker, K.L., and M.P. Gillingham. 1987. Estimating thermal environments for mule deer. Ministries of Environment and Forests, WHR-24, Victoria, B.C.

Parker, K.L., and C.T. Robbins. 1984. Thermoregulation in mule deer and elk. Can. J. Zool. 62:1409-1422.

Parker, K.L., and C.T. Robbins. 1985. Thermoregulation in ungulates. p. 161-213 In: R.J. Hudson and R.G. White (eds), Bioenergetics of wild herbivores. CRC Press, Boca Raton, Fla.

Renecker, L.A., and R.J. Hudson. 1986. Seasonal energy expenditures and thermoregulatory responses of moose. Can. J. Zool. 64:322-327.

Renecker, L.A., R.J. Hudson, M.K. Chriatophereen, and C. Arelin. 1978. Effect of posture, feeding, low temperature, and wind on energy expenditures of moose calves. Proc. of the 14th N. Amer. Moose Conf. Workshop. 14:126-140. 
Thomas, J.W., J. Black, Jr., R.J. Scherdinger, and R.J. Pederien. 1979. Deer and elk. p. 104-127 ln: J.W. Thomas (ed), Wildlife habitats in managed forests: the Blue Mountains of Oregon and Washington. USDA Forest Serv., Agr Handbook 553.

Webster, A.J.F. 1974. Physiological effects of cold exposure. p. 33-69. In: D. Robertshaw (ed), Environmental physiology: Physiology Series One, Vol. 7. Butterworths, London.

Wealey, D.E., K.L. Knox, and J.G. Nasy. 1973. Energy metabolism of pronghorn antelopes. J. Wildl. Manage. 37:563-573.

Wesley, M.L., and R.C. Lpachutz. 1976. An experimental study of the effects of aerosols on diffuse and direct solar radiation received during the summer near Chicago. Atmos. Environ. 10:981-987.

Appendix 1: Calculation of standard operative temperature $\left(T_{m}\right)$ experienced by an animal

$$
T_{e s}=T_{b}-\frac{r_{H b s}+r_{e}}{r_{H b}+r_{e}} \cdot\left(T_{b}-T_{e}\right)
$$

(Bakken 1981)

where:

$$
T_{e}=T_{a}+\frac{r_{e}\left(R_{\text {bbs }}-\epsilon_{s} \sigma T_{2}^{4}\right)}{\rho c_{p}}
$$

(Campbell 1977:94)

and: $T_{\mathrm{e}} \quad=$ operative temperature $\left({ }^{\circ} \mathrm{C}\right)$

$T_{\mathrm{a}} \quad=$ air temperature $\left({ }^{\circ} \mathrm{C}\right)$

$r_{e}=$ equivalent resistance to convective and radiative heat transfer $\left(\mathbf{s} \cdot \mathrm{m}^{-1}\right)$

$\mathbf{R}_{\mathrm{abs}}=$ solar (SW) and thermal radiation (LW) absorbed by the animal $\left(\mathbf{W} \cdot \mathbf{m}^{-2}\right)$

$\epsilon_{8} \sigma \mathrm{T}_{\mathrm{a}}^{4}=$ thermal radiation emitted by the animal $\left(\mathrm{W} \cdot \mathrm{m}^{-2}\right)$

$\rho \mathrm{c}_{\mathrm{p}}=$ density of the air $(\rho) \cdot$ specific heat $\left(\mathrm{c}_{\mathrm{p}}\right)\left(1200 \mathrm{~J} \cdot \mathrm{m}^{-3} \cdot{ }^{\circ} \mathrm{K}^{-1}\right.$; Campbell 1977:9)

$T_{\mathrm{b}} \quad=$ body temperature $\left({ }^{\circ} \mathrm{C}\right)$

$\mathrm{r}_{\mathrm{Hb}} \quad=$ whole body thermal resistance $\left(\mathrm{s} \cdot \mathrm{m}^{-1}\right)$

$\mathrm{r}_{\mathrm{Hb}}=\mathrm{r}_{\mathrm{Hb}}$ under low convective conditions $\left(\mathrm{u} \leq 1 \mathrm{~m} \cdot \mathrm{s}^{-1}\right)$

$r_{e}=r_{e}$ under low convective conditions $\left(u \leq 1 \mathrm{~m} \cdot \mathrm{s}^{-1}\right)$

SHORT-WAVE RADIATION ABSORBED (SW):

$$
S W=a_{d}\left(A_{p} / A \cdot S_{p}+0.5 \cdot S_{d}+0.5 \cdot S W G R\right)
$$

where: $a_{4} \quad=$ absorptivity to shortwave radiation $(0.70$ in summer, 0.80 in winter, W. P. Porter pers. comm.)

$\mathrm{Ap} / \mathrm{A}=$ ratio of shadow area on a surface perpendicular to the solar beam (0.3; Campbell 1977:80)

$S_{\mathrm{p}} \quad=$ direct beam radiation on surface perpendicular to beam $\left(\mathrm{W} \cdot \mathrm{m}^{-\mathbf{2}}\right)$

$S_{d} \quad=$ diffuse short-wave radiation $\left(W \cdot \mathrm{m}^{-2}\right)$

SWGR $=$ reflected short-wave radiation from ground $\left(\mathrm{W} \cdot \mathrm{m}^{-2}\right)$

Incident Short-Wave Radiation

$$
\begin{aligned}
& \mathrm{S}_{\mathrm{p}}=\mathrm{S}_{\mathrm{b}} / \sin \theta \\
& \mathrm{S}_{\mathrm{d}}=\alpha\left(\beta \mathrm{S}_{\mathrm{pos}} \sin \theta-\mathrm{S}_{\mathrm{T}}\right) /(1-\alpha) \\
& \mathrm{S}_{\mathrm{d}}=\mathrm{S}_{\mathrm{T}} \text { for } \mathrm{S}_{\mathrm{T}}<0.5 \cdot \mathrm{S}_{\mathrm{po}} \sin \theta \\
& \mathrm{S}_{\mathrm{T}}=\mathrm{S}_{\mathrm{b}}+\mathrm{S}_{\mathrm{d}}
\end{aligned}
$$

where: $S_{\mathrm{b}}=$ incident beam radiation $\left(\mathrm{W} \cdot \mathrm{m}^{-2}\right)$

$S_{\mathbf{T}}=$ total incident short-wave radiation (beam + diffuse; $W$. $\mathrm{m}^{-2}$ ).

$S_{p o}=$ solar constant of extra-terrestrial flux density $(1360 \mathrm{~W}$. $\mathrm{m}^{-2}$; Campbell 1977:55)

$\alpha=$ scalar incorporating differences between potential direct radiation and solar constant $(0.64$; Wesley and Lipschutz 1976)

$\beta=$ scalar incorporating absorption of solar beam by moisture and ozone (0.79; Wesley and Lipschutz 1976)

$\theta=$ solar angle (degrees);

and: TLA = TD + LONGC + EQ (Paltridge and Platt 1976)

EQ $\quad=0.000075+0.001868 \cdot \cos (\phi)-0.032077 \cdot \sin (\phi)-$ $0.014615 \cdot \cos (2 \phi)-0.040849 \cdot \sin (2 \phi)$

LONGC $=+4 \mathrm{~min}$ for each ${ }^{\circ} \mathrm{E}$ and $-4 \mathrm{~min}$ for each ${ }^{\circ} \mathrm{W}$ of standard meridian $\begin{aligned}= & 0.006918-0.399912 \cdot \cos (\phi)+0.070257 \cdot \sin (\phi)- \\ & 0.006758 \cdot \cos (2 \phi)+0.000907 \cdot \sin (2 \phi)-0.002697 .\end{aligned}$ $\cos (3 \phi)+0.001480 \cdot \sin (3 \phi)$

$\sin \theta=\sin \delta+\sin ($ lat $)+\cos \delta \cos ($ lat $) \cdot \cos (15$ (TLA-TSN) $)$

where: TSN = time of solar noon

TLA = local apparent time

TD = clock time

LONGC = longitudinal correction

EQ $\quad=$ equation of time

$\delta=$ solar declination

lat $\quad=$ latitude

$\phi \quad=2 \cdot \pi \cdot \mathrm{J} / 365$

$\mathrm{J} \quad=$ Julian day

Reflected short-wave radiation from ground

$$
S W G R=\text { Albedo } \cdot S_{T}
$$

where: Albedo $=0.8$ for snow; 0.20 for typical field; 0.26 for matted grass (Lowry 1969:133)

LONG-WAVE RADIATION ABSORBED (LW):

$$
\mathrm{LW}=\mathrm{a}_{\mathrm{L}}\left(0.5 \cdot \epsilon_{2 \mathrm{ky}} \sigma \mathrm{T}_{\mathrm{a}}^{4}+0.5 \cdot \epsilon_{\mathrm{er}} \sigma \mathrm{T}_{\mathrm{a}}^{4}\right)
$$

where: $\sigma=$ Stephan Boltzman constant $\left(5.67 \cdot 10^{-8} \mathrm{~W} \cdot \mathrm{m}^{-2} \cdot \mathrm{K}^{-4}\right)$

$a_{L}=$ absorptivity to long-wave radiation (1.0 for caribou; Monteith 1973)

$\epsilon_{2 k y}=$ emissivity of sky (see Moen 1968:340)

$\epsilon_{\mathrm{gr}}=$ emissivity of ground (0.97; Parker unpubl.)

$\mathrm{T}_{\mathrm{a}}{ }^{4}=$ air temperature in ${ }^{\circ} \mathrm{K}\left(\left(\mathrm{T}_{\mathrm{a}}+273\right)^{4}\right)$

LONG-WAVE RADIATION EMITTED FROM ANIMAL $\left(\mathbf{L}_{\infty}\right)$ :

$$
\mathrm{L}_{\mathrm{oc}}=\epsilon_{\mathrm{a}} \sigma \mathrm{T}_{\mathrm{a}}^{4}
$$

where: $\epsilon_{3}=$ emissivity of surface (1.0 for caribou, Monteith 1973)

$\mathrm{T}_{\mathrm{a}}{ }^{4}=$ air temperature in ${ }^{\circ} \mathrm{K}\left(\left(\mathrm{T}_{\mathbf{a}}+273^{\circ}\right)^{4}\right)$

THERMAL RESISTANCE BETWEEN OUTER SURFACE AND ENVIRONMENT $\left(r_{e}\right)$ :

$$
\begin{aligned}
& 1 / r_{\mathrm{e}}=1 / \mathrm{r}_{\mathrm{Hz}}+1 / \mathrm{r}_{\mathrm{r}} \\
& r_{\mathrm{r}}=\rho \mathrm{c}_{\mathrm{p}} / 4 \epsilon_{\mathrm{g}} \sigma \mathrm{T}_{\mathrm{a}}{ }^{3} \\
& r_{\mathrm{Ha}}=307 \sqrt{\mathrm{d} / \mathrm{u}} \cdot 0.7 \\
& \mathrm{r}_{\text {Hasun }}=840\left[\mathrm{~d} /\left(\mathrm{T}_{\mathrm{a}(\mathrm{sun})}-\mathrm{T}_{\mathrm{a}}\right)\right]^{0.25} \\
& r_{\text {Hanhade }}=840\left[\mathrm{~d} /\left(\mathrm{T}_{\text {(chade })}-\mathrm{T}_{\mathrm{2}}\right)\right]^{0.25} \\
& Z_{p d}=0.77 \mathrm{~h} \\
& \mathrm{Z}_{\mathrm{m}}=0.13 \mathrm{~h} \\
& u^{*}=u(k) /\left(\ln \left(Z_{v}+Z_{m}-Z_{p d}\right) / Z_{m}\right) \\
& u_{\mathrm{a}}=\left(\mathrm{u}^{*} / \mathrm{k}\right) \cdot \ln \left(\left(\mathrm{Z}-\mathrm{Z}_{\mathrm{m}}-\mathrm{Z}_{\mathrm{pd}}\right) / \mathrm{Z}_{\mathrm{m}}\right)
\end{aligned}
$$

where: $r_{e} \quad=$ thermal resistance of skin and pelage to heat flow

$r_{r} \quad=$ resistance to long-wave radiative transfer

$r_{\mathrm{H}}=$ resistance to forced convective heat transfer

$\mathrm{r}_{\mathrm{H} \text { soun }}=$ resistance to free convection in sun

$\mathrm{r}_{\text {Hestede }}=$ resistance to free convection in shade

d = characteristic dimension of animal [(length + width)/ 2; Parker 1987)

$0.7=$ turbulence factor (Campbell 1981)

$\mathrm{Z}_{\mathrm{pd}} \quad=$ zero plane of displacement or distance from height zero to average height of heat exchange (Campbell 1977:38)

h = average 'crop' height (grass, shrubs, snow)

$\mathrm{Z}_{\mathrm{m}} \quad$ = momentum roughness parameter for uniform surfaces or length of drag at momentum exchange surfaces (Campbell 1977:39; see Bussinger (1975) for more complex surfaces)

$\mathrm{Z}_{\mathrm{u}} \quad=$ height of wind measurement (standard is $3 \mathrm{~m}$ )

$\mathrm{k} \quad=$ von Karman's constant (0.4; Campbell 1977:38)

$\mathrm{Z} \quad=$ animal height (assumed to be rib cage; Parker 1987)

$\mathrm{u} \quad=$ wind speed, $\mathrm{m} \cdot \mathrm{s}^{-1}$

$u^{*} \quad=$ friction velocity

$\mathrm{u}_{\mathrm{a}} \quad=$ wind speed extrapolated to animal height $\left(\mathrm{m} \cdot \mathrm{s}^{-1}\right)$

$T_{3} \quad=$ skin temperature (from Parker and Robbins 1984) 
BODY TEMPERATURE $\left(\mathrm{T}_{\mathrm{b}}\right)$ :

$\mathrm{T}_{\mathrm{b}}=37.95+6.0 /\left(1+\mathrm{e}^{-0.103(\mathrm{Te}-53.65)}\right.$ ) in winter (from Parker and Robbins 1984)

$T_{b}=38.18+6.0 /\left(1+e^{-0.153(T e-51.01)}\right)$ in summer (from Parker and Robbins 1984)

WHOLE BODY THERMAL RESISTANCE $\left(\mathbf{R}_{\text {HRe }}\right)$

$\mathrm{r}_{\mathrm{HBs}}=857.9-29.9 \mathrm{~T}_{\mathrm{e}}-0.19 \mathrm{~T}_{\mathrm{e}}{ }^{2}+0.006 \mathrm{~T}_{e}{ }^{3}$ in winter (from Parker and Robbins 1984)

$\mathrm{r}_{\mathrm{HBs}}=603.29-4.62 \mathrm{~T}_{\mathrm{c}}-0.30 \mathrm{~T}_{\mathrm{e}}{ }^{2}$ in summer (from Parker and Robbins 1984)

$\mathbf{r}_{\mathrm{He}}=\mathbf{r}_{\mathrm{HBs}}-\mathrm{r}_{\mathrm{Ht}}$

$r_{H_{2}}=63.95-5.58 T_{e}$ in winter (Webster 1974)

$r_{\mathrm{Ht}}=204.43-6.49 \mathrm{~T}_{\mathrm{e}}$ in summer (Webster 1974) where: $r_{\mathrm{Hes}}=$ thermal resistance of the coat under low convective conditions $(\mathrm{u} \leq 1 \mathrm{~m} / \mathrm{s})$

$\mathbf{r}_{\mathrm{Ht}}=$ thermal resistance of the tissue

WHOLE BODY THERMAL RESISTANCE TO OUTDOOR WIND SPEEDS:

$\mathrm{r}_{\mathrm{Hb}}=\mathrm{r}_{\mathrm{Ht}}+\mathrm{r}_{\mathrm{Hc}}$

$r_{\mathrm{Hc}}=r_{\mathrm{Hed}} /\left(1.0+0.08 u_{\mathrm{n}}\right)($ Campbell et al. 1980)

where: $r_{H c}=$ thermal resistance of the coat under outdoor wind speeds

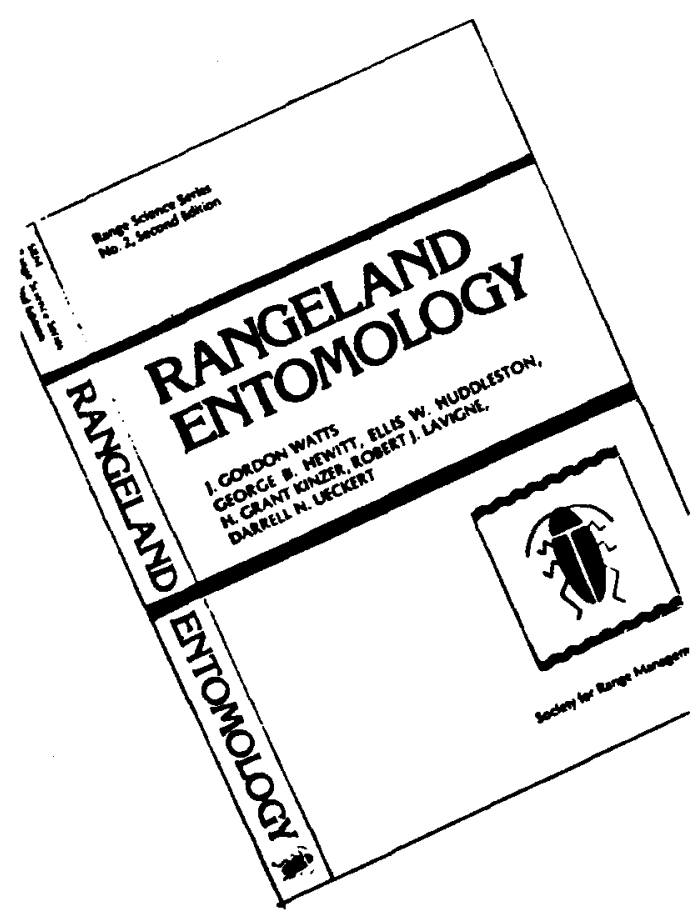

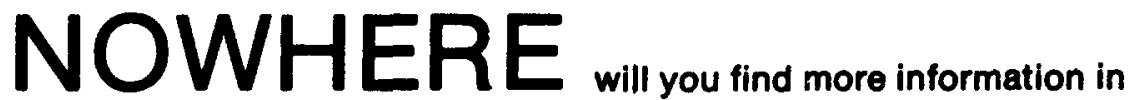
one place on this increasingly important subject. A greater variety of uses for rangeland, a growing world population, plus deeper concerns for the environment make knowledge about insect grazers of rangeland ever-more critical. Written to be understood by the average reader, RANGELAND ENTOMOLOGY still contains the extensive bibliography, lists of both common and scientific names, and suggestions for needed research to make it a valuable addition to the scientist's library. The second edition, edited by J. Gordon Watts, has been expanded to over 300 pages with state-of-the-art information on insect friends and foes of rangeland plants and animals and the role of integrated pest management.

Range Science Series No. 2, Second Edition, will be available from the Society for Range Management by February 1990. 